\title{
OSTEOID OSTEOMA OF VERTEBRAE
}

\author{
W. T. Mustard, Toronto, Canada, and F. W. DuVal, * Winnipeg, Canada
}

Osteoid osteoma is no longer a rare lesion. The two cases reported here did however present in an unusual fashion and did pose difficult diagnostic problems.

\section{CASE REPORTS}

Case 1-An eight-year-old boy was first seen by the senior author in November 1949, after poliomyelitis in July of that year. His upper and lower extremities and abdominal musculature were normal, but he stood with a decided cervical scoliosis, convex to the right. He complained of intermittent pain in the back of the left shoulder, occasionally radiating down the back of the left arm to the wrist, but not into the hand. The pain was present only at night. A radiograph of the left shoulder did not show any abnormality. During the next five months there was a gradual increase in the curve, and the pain in the shoulder increased. Spot radiographs of the upper thoracic and lower cervical regions revealed an area of increased density in the first thoracic vertebra, and osteoid osteoma was suspected (Fig. 1). A plaster jacket was applied for about a month without relief of the pain.

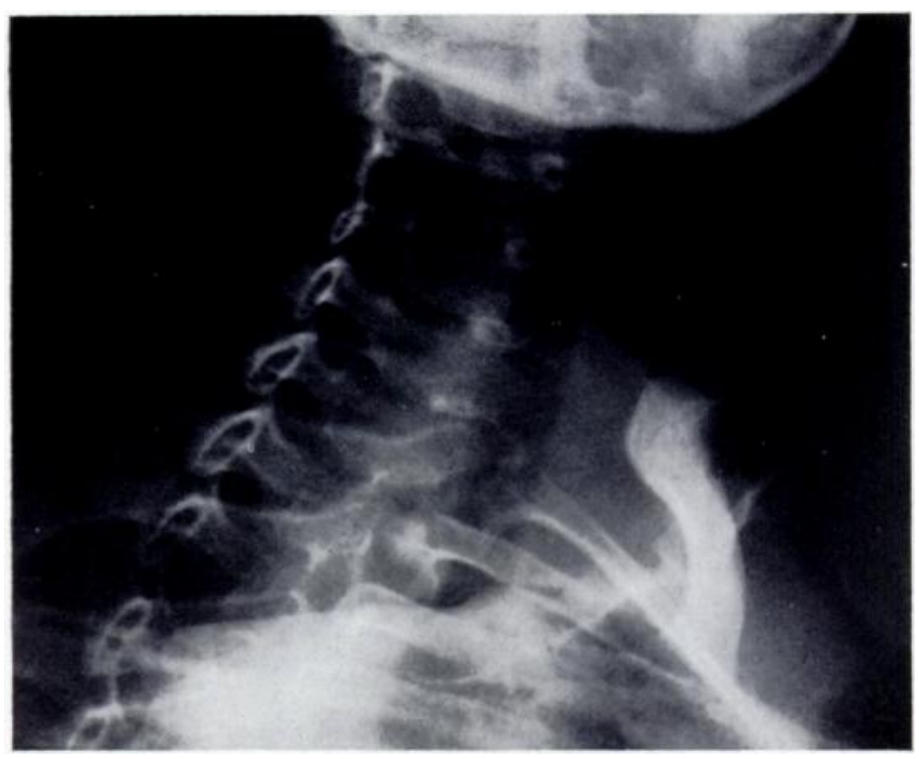

FiG. 1

Case 1 -The zone of increased density in $T .1$ vertebra.

On his admission to the Hospital for Sick Children (Toronto), a three-foot standing film of the spine revealed that the angle of the primary curve had increased from 12 to 22 degrees (Fig. 2). A point of tenderness was present just above and medial to the superior angle of the left scapula, and pain was elicited there by applying pressure to the top of the head and forcing it to the left. However, the radiating pain could not be reproduced. Neither sensory nor reflex change was noted in the arm, but there was marked supraclavicular and infraclavicular wasting on the left. The sedimentation rate was 18 millimetres in the first hour

\footnotetext{
*Attending Surgeon, Winnipeg Children's Hospital.
} 


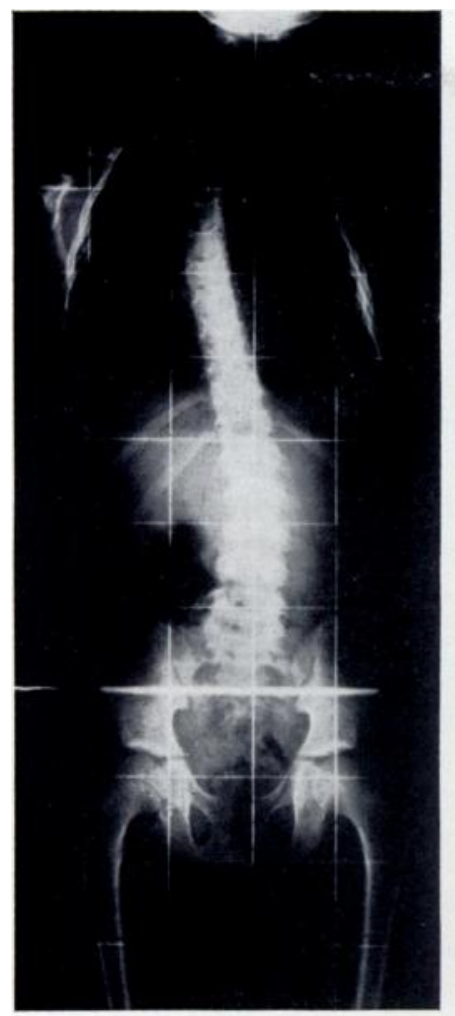

FIG. 2

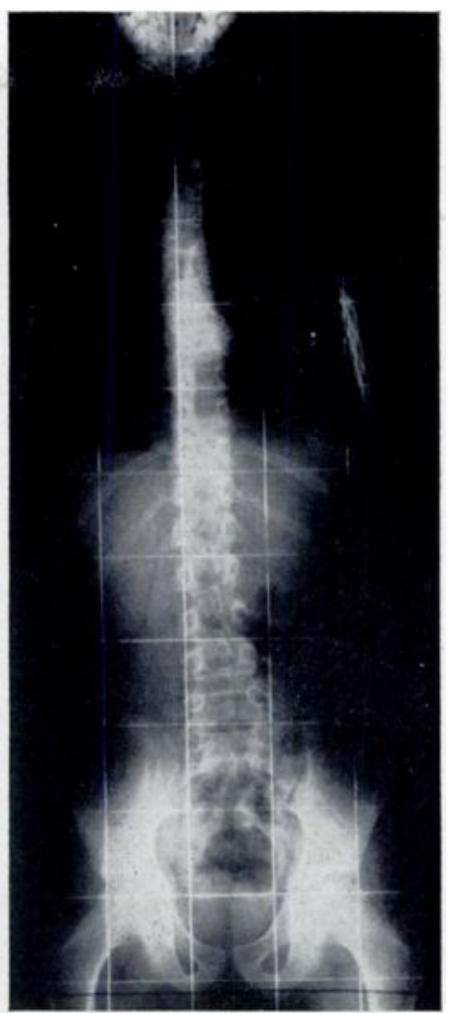

FIG. 3

Case 1. Figure 2-Three-foot standing radiograph of the spine with marked scoliosis convex to the right in upper thoracic and cervical regions. Figure 3-Three-foot standing radiograph of the spine six years after removal of the lesion.

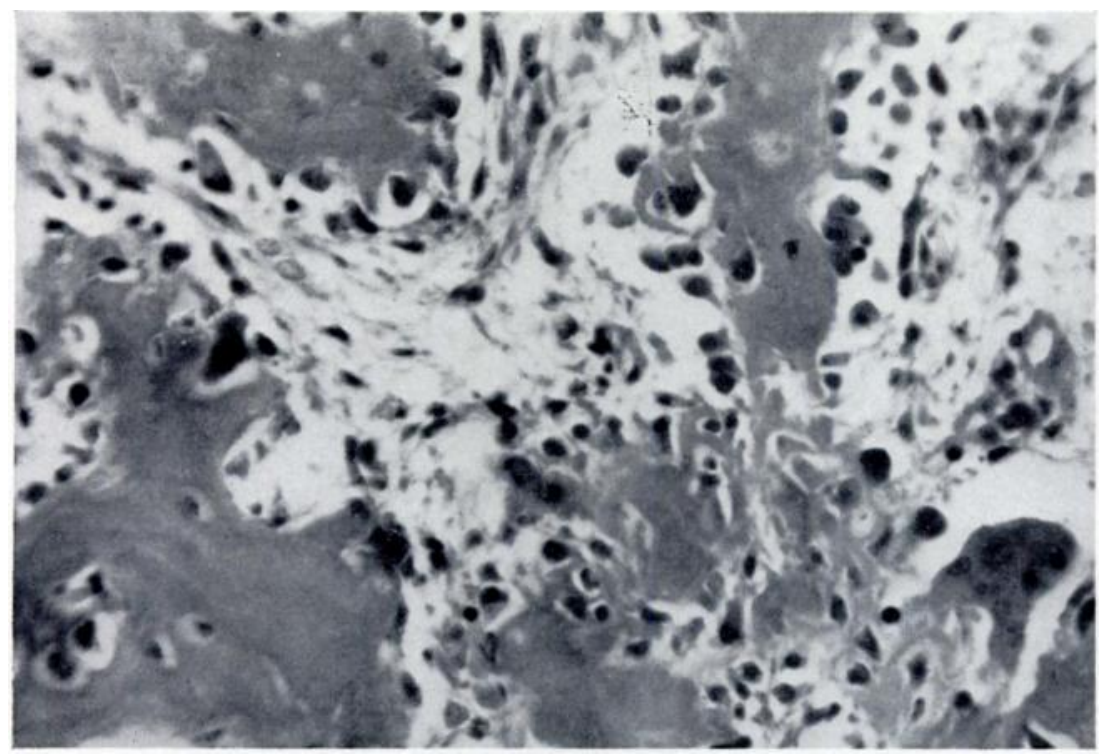

Fig. 4

Case 1-Photomicrograph of a section of the specimen. $(\times 200$. $)$

VOL. $41 \mathrm{~B}$, NO. 1, FEBRUARY 1959 
(Wintrobe), the white cell count 10,500 per cubic millimetre and haemoglobin $13 \cdot 2$ grammes per cent.

Operation-In April 1950 the lamina on the left of T.1 vertebra was exposed. This was curetted, and a discrete calcified nidus was found and removed (Fig. 4). The surrounding sclerotic process involved neural arch, body and articular process. Because of the destruction incident to removal of the nidus, an autogenous tibial bone graft was placed from C.7 to T.2 on the right side.

Progress-The boy was last seen in March 1956, six and a half years later. He still complained of occasional pain in the back of the left arm, but this was believed to be a sequela of the operation. Certainly the local tenderness, wasting and the night pain were no longer present, and a three-foot standing radiograph of the spinal column showed that the scoliosis had completely disappeared (Fig. 3).

Case 2-A ten-year-old boy was admitted to the Hospital for Sick Children (Toronto) in August 1950, with intermittent lumbar pain of six months' duration which was present on his arising in the morning and lasted for two or three hours each day. There was no complaint of pain at night. The pain was increased by forward flexion of the body, but not aggravated by exercise. There were no previous illnesses.

Examination revealed a healthy, well nourished boy. The head and neck, chest, abdomen and extremities were normal. The back showed marked spasm of the semispinalis muscle on the right in the lumbar region, with moderate scoliosis convex to the left extending from T.9 to L.4. There was reduced lateral flexion to the left and the normal lumbar curve had

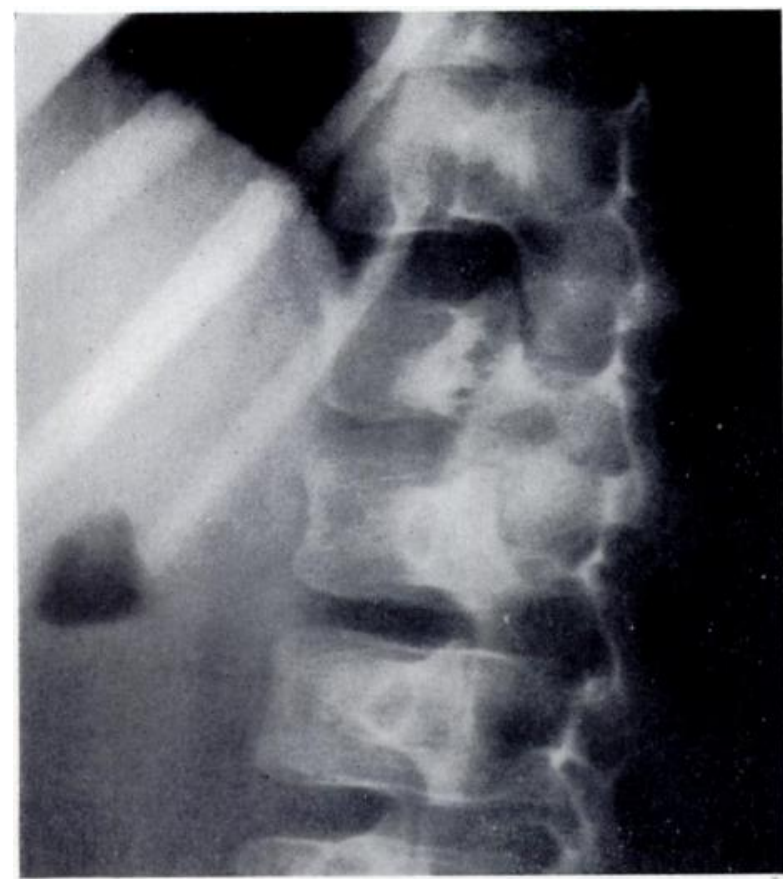

Fig. 5

Case 2-Spot radiographs of lumbar region with area of decreased density in region of articular facet of L.1. disappeared (Fig. 6). Spot films showed a circumscribed area of decreased density 1.5 centimetres in diameter in the first lumbar vertebral body, at the junction of the right neural arch and inferior articular process (Fig. 5).

Operation-The lesion in the lamina of L.1 was exposed by retracting the muscle mass on the right. A small piece of the muscle adjacent to the lesion was excised and submitted for frozen section. This was shown to be normal muscle with some fatty degeneration. The tumour wascuretted, and was found to invade the articular facet, body and lamina. These and the spinous process were removed (Fig. 8). Bone flakes were turned down on the spinous processes of T.12 and L.2 vertebrae and an autogenous tibial bone graft was applied on the right side. The spinous processes of T.12 and L.2 vertebrae were wired together. A short body jacket was retained for three months.

Progress-Seen in March 1956 the patient had no complaint of back pain; clinically the back was straight and a three-foot standing film of the spine showed no curve (Fig. 7).

Histological examination-The specimens in both cases have been examined by $\mathrm{Dr} \mathrm{W}$. L. Donohue, of the Hospital for Sick Children (Toronto), who confirmed the diagnosis of osteoid osteoma (Figs. 4 and 8). 


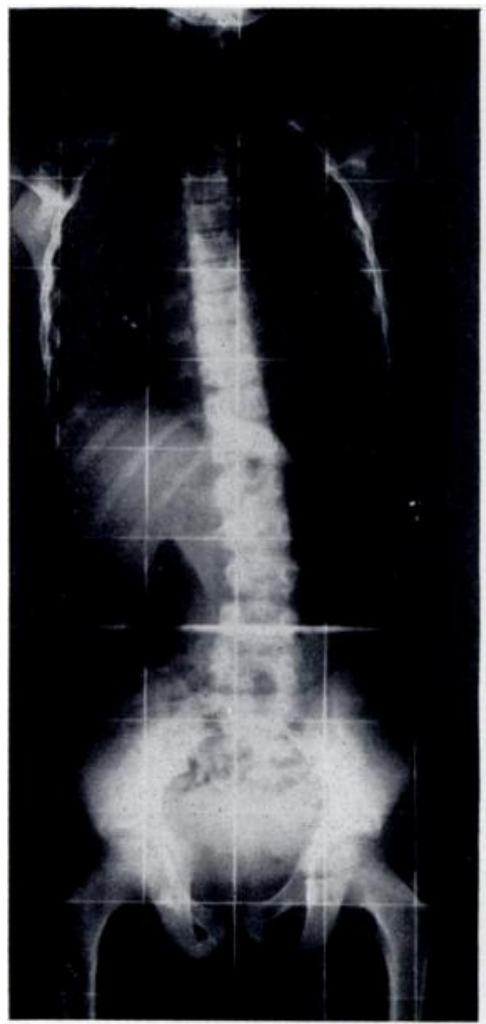

FIG. 6

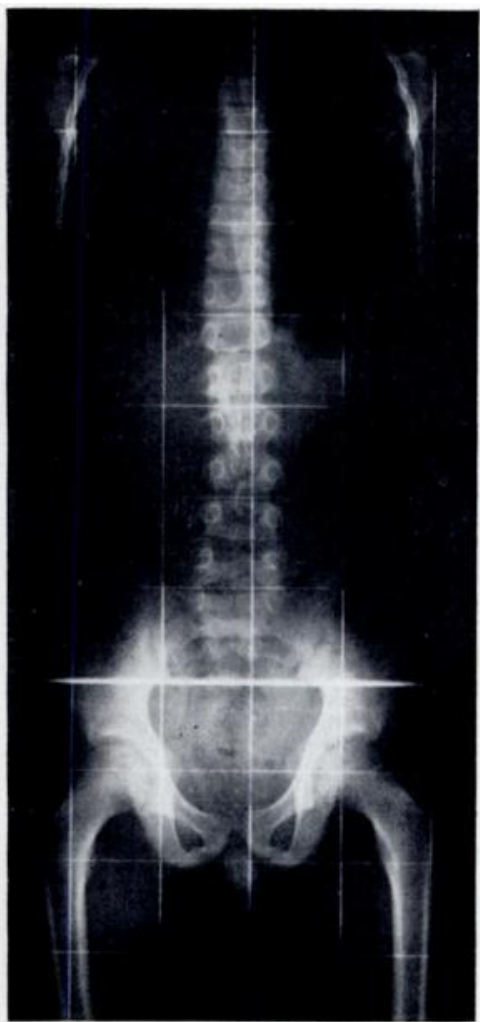

FIG. 7

Case 2. Figure 6-Three-foot standing radiograph of the spine with scoliosis convex to the left. Figure 7-Three-foot standing film six years after operation.

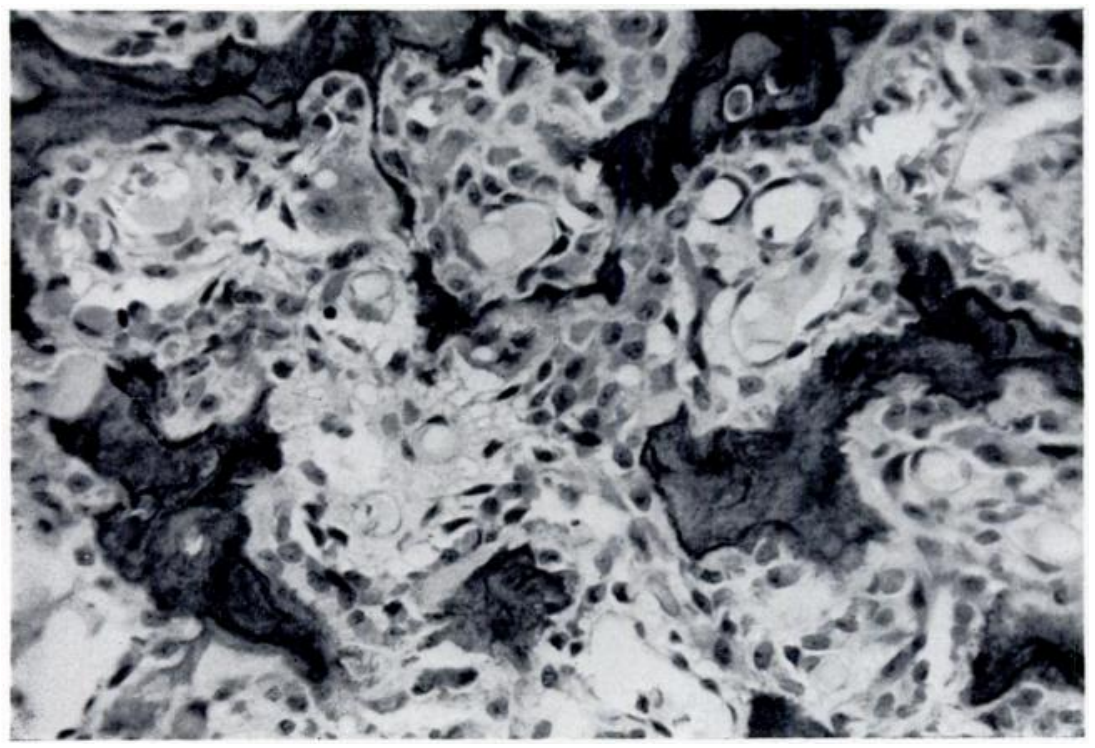

Fig. 8

Case 2-Photomicrograph of a section of the specimen. $(\times 200$.) 


\section{DISCUSSION}

Both cases presented similar difficult diagnostic problems: 1) the appearance of a scoliosis, one apparently an idiopathic curve, and the other probably paralytic in view of the recent history of poliomyelitis and the apparent progression of the curve over a period of five months; 2 ) the definite and marked history of pain; 3) the gradual increase in severity of symptoms during six months; 4) the difficult radiological identification of the lesion in the vertebra; 5) the similar position of both lesions, each involving vertebral body, neural arch and articular facet; 6) relief of symptoms by curettage and removal of the lesion; and 7) disappearance of the scoliotic curve after removal of the lesion.

Of more than 280 cases of osteoid osteoma described since 1935 (Jaffe), only twenty-five have been in a vertebra (Fagerberg and Rudström 1953, Dahlin and Johnson 1954). Sherman (1947) alone has written of scoliosis as a presenting sign, although without detail.

Dahlin and Johnson (1954), describing four cases involving a vertebra, did not refer to scoliosis although a reproduction of a radiograph did show a mild scoliosis in one patient. Two of the lesions involved body, lateral mass and a facet, as in our two cases. Few detailed reports are to be found. Osteoid osteoma has been identified in the spinous process, and Dockerty, Ghormley and Jackson (1951) described one in the pedicle of the third lumbar vertebra.

There has been much speculation about the natural history of the disease. Sherman (1947a) discussed a case of Dr D. B. Phemister. An eighteen-year-old boy, seen before the publication of Jaffe's original description, had refused operation for a tibial lesion that radiologically would now be considered an osteoid osteoma. He returned twenty-four years later with a history of spontaneous remission of pain seven years after the onset, and a radiograph now showed no evidence of a nidus although the typical sclerotic bone persisted. Dockerty et al. (1951) described a seven-year-old girl with the clinical and radiological features of an osteoid osteoma of the femur. Her symptoms regressed in two years, and seven years later the radiograph appeared normal. Moberg (1951), considering the natural course of osteoid osteoma, illustrated his thesis of spontaneous regression by a metacarpal lesion that ceased to cause symptoms after two years. Additional indirect evidence for spontaneous remission of the lesion is that it seldom occurs in persons over thirty years of age.

After Jaffe had persuaded most writers that osteoid osteoma was a true tumour in spite of its self-limitation, three further cases have been reported that appear to revive the inflammatory theory. They were all adjacent to joints-two in the femoral neck, and one in the humerus in the synovium. The picture was identical, both grossly and microscopically, with that of rheumatoid arthritis (Sherman 1947b, Massachusetts 1955).

\section{SUMMARY}

1. Two cases of osteoid osteoma of the vertebrae are presented.

2. The unusual clinical histories and findings are reported in detail.

\section{REFERENCES}

Case Records of the Massachusetts General Hospital (1955): Osteoid Osteoma of Neck of Femur. New England Journal of Medicine, 253, 701.

Dahlin, D. C., and Johnson, E. W., Jun. (1954): Giant Osteoid Osteoma. Journal of Bone and Joint Surgery, 36-A, 559.

Dockerty, M. B., Ghormley, R. K., and Jackson, A. E. (1951): Osteoid Osteoma. Annals of Surgery, $133,77$. Fagerberg, S., and Rudström, P. (1953): Osteoid Osteoma of a Vertebral Arch. Acta Radiologica, 40, 383. JAFFE, H. L. (1935): “ Osteoid-Osteoma.” Archives of Surgery, 31, 709.

Moberg, E. (1951): The Natural Course of Osteoid Osteoma. Journal of Bone and Joint Surgery, 33-A, 166. Sherman, M. S. (1947a): Osteoid Osteoma. Journal of Bone and Joint Surgery, 29, 918.

Sherman, M. S. (1947b): Osteoid Osteoma Associated with Changes in Adjacent Joint. Journal of Bone and Joint Surgery, 29, 483. 